\title{
Leprosy and Primary Health Care: Tanzania
}

\author{
S J NKINDA \\ Ministry of Health, P O Box 9083, Dar es Salaam, Tanzania
}

Received for publication 2 September 1981

\section{Introduction}

Primary Health Care (PHC) as defined at the Alma-Ata Conference of $1978,{ }^{1}$ will deal with at least the following 8 functions: education of the community on major health problems; promotion of proper nutrition; adequate supply of safe water and basic sanitation; maternal and child health and family planning; immunizations against major infectious diseases; prevention and control of endemic diseases; treatment of common diseases and injuries; and provision of essential drugs.

Leprosy is one of the endemic diseases in many developing countries. It therefore, falls under the scope of PHC. The Fifth Report of the WHO Expert Committee on Leprosy $(1974)^{2}$ observed that the PHC approach may well be appropriate for the introduction of leprosy control. Sansarricq ${ }^{3}$ considered PHC to be best adapted to obtain patient compliance for prolonged chemotherapy. Participants from African countries attending a WHO consultation on integration of leprosy and tuberculosis services ${ }^{4}$ proposed PHC as one of the solutions for improving efficiency, coverage and benefits to the people. Buchmann ${ }^{5}$ listed several benefits of integrating leprosy control into PHC.

The intention of this paper is to examine the pre-requisites to be met before PHC can make its maximum contribution to leprosy control, to consider how the objectives of leprosy control can be achieved through PHC and, finally, to show by examples from Tanzania that leprosy can be tackled by the PHC approach.

\section{Pre-requisites for implementing PHC}

Political commitment to $\mathrm{PHC}$ is a top priority requirement and it is the single most important factor for overcoming obstacles to PHC. ${ }^{6}$ This determination 
must be seen in practical terms. The Ministry of Health has to take the initiative to mobilize the machinery that will enact the necessary legislation and make the required budgetary allocations.

Community participation is the next high priority requirement if $\mathrm{PHC}$ is to succeed in bringing about improvements in health. Better health cannot be achieved if health care remains only as services brought to the community by visiting health workers. The people must accept greater responsibility for their health. The community must develop self-reliance. Active participation in planning and implementation of health measures by the community is essential.

Another priority requirement is the selection and proper training of the Village Health Worker (VHW). The community should select candidate(s) for training. The training should be of short duration and conducted under conditions which are similar to those of the village. Thus, it is better to train them at a rural health centre rather than in a big regional or consultant hospital. The training should focus on enabling the workers to perform carefully selected tasks. Modern educational methods emphasize the importance of analysis of tasks and functions which will indicate the attitudes, knowledge and skills to be imparted to the trainees. ${ }^{7}$

Other preconditions to be considered include ensuring that drugs and equipment are available without interruptions, a functioning referral system is present to cater for complications of the disease and last but not least effective supervision is provided.

\section{PHC - an unavoidable choice in Tanzania}

In the words of Professor Chuke ${ }^{8}$ 'the greatest priority in health services in Africa is the extension of organized health services to such an extent that every citizen is within walking distance of a health facility'. Since 1971-72 the Government of Tanzania has been doing this for the majority (90\%) of the people in rural areas. A change in emphasis from hospital-urban-based health care to rural health services was made in financial allocations, manpower training and building of rural infrastructures.

The health facilities which provide PHC are, in general, organized on a 4level system ${ }^{9}$ and specific examples from Tanzania ${ }^{10}$ include the following health units, shown in Table 1.

1 Village health post manned by VHW serving 1,500 to 2,000 inhabitants.

2 Rural dispensary with about 3 health workers who serve about 5 villages (that is, a ward) or a population of up to 10,000 people.

3 Rural health centre staffed by $8-10$ workers. It is part of the referral system for about five dispensaries and several village health posts and is intended to serve about 50,000 people (that is, a division). 
Table 1. Hierachy and administrative structure of PHC in relation to political set up, Tanzania

\begin{tabular}{clllc}
\hline $\begin{array}{c}\text { Administrative } \\
\text { level }\end{array}$ & Political head & Health facility & $\begin{array}{c}\text { Personnel } \\
\text { in Charge }\end{array}$ & $\begin{array}{c}\text { Population } \\
\text { Served }\end{array}$ \\
\hline Village & Village & Village & VHW & 1,000 to \\
& Chairman & Health Post & & 2,000 \\
Ward & Ward & Rural & Rural & 5,000 to \\
& Secretary & Dispensary & Medical Aid & 10,000 \\
Division & Divisional & Rural & Medical & 50,000 \\
& Secretary & Health Centre & Assistant & and Over \\
District & Area & District & District & $\pm 250,000$ \\
& Commissioner & Hospital & Medical Officer \\
\hline
\end{tabular}

4 A district hospital (also called primary level hospital) manned by professional medical, nursing and other health personnel and may cater for about 250,000 people.

The target for rural dispensaries was almost achieved by 1980 with a ratio of 1 dispensary per 6,700 inhabitants and for rural health centres the figures stood at 1 per 70,000 inhabitants - still far away from the target of 1 per 50,000 people. $^{11}$

Conventional health manpower, including auxiliaries, have increased. In 1980 Tanzania had 1 doctor per 20,000 inhabitants compared with 1 per 28,000 in 1972 . For the auxiliary cadres which are the pillars of rural health services, rural medical aids have increased from 1 per 30,000 inhabitants in 1972 to 1 per 8,700 people in $1980 .{ }^{11}$

The health workers for PHC in Tanzania include medical assistants, rural medical aids, community nurses including $\mathrm{MCH}$ aids, health officers (sanitarians) and health auxiliaries. The training for these cadres ranges from 18 months to 3 years. In order to cater for communities without any health facility, the country will have to resort to VHW who can be trained for shorter periods and at much less cost than the mentioned cadres.

As a result of this deliberate effort to expand health infrastructures for the majority of the people and the villagization programme whereby scattered rural people were brought together into village communities, the people now live nearer to health facilities than in the past. Ninety-two per cent of the people are within $10 \mathrm{~km}$ of a health facility, and $70 \%$ live within the acceptable walking limit of $5 \mathrm{~km}$ or 1 hour from a health unit. However, the distribution of the health units is such that only $45 \%$ of the people have a health unit near their place of residence. ${ }^{11}$ Because leprosy still needs long-term treatment, the nearer the treatment points are the better. Regularity or frequency of clinic attendance is very much influenced by the distance the patient has to travel. King ${ }^{12}$ showed that on average outpatient attendance per person drops by half for about every increase of $3 \mathrm{~km}$ between the patient's home and the health units. Even the relatively regular lepromatous leprosy patient, having to 
walk $20 \mathrm{~km}$ or more on the return journey between home and health unit on clinic days for 10 years is a taxing undertaking. This is still the lot of some communities in Tanzania. PHC holds better prospects for patients from these villages.

Several of the prerequisites for implementing PHC exist in Tanzania. Selfhelp is an accepted basic political concept which requires involvement of the people in the preparation and execution of plans. There is an organizational framework which favours active community participation. Each registered village has a village council made up of several committees, one being responsible for health, education, culture and social welfare. Through such organs the people can identify their requirements, agree on their priorities and decide which activities they can be self-reliant in implementing and those which they should seek government help for. More than $66 \%$ of villagers expressed willingness to contribute labour, materials and money to construct health facilities. ${ }^{13}$

The country has had some experience with VHW. In order to provide health care to villages without health facilities, village health posts were started at the initiative of district authorities and villagers. More than 2,000 VHW have provided health care in this way. Their functions included treatment of minor ailments, environmental sanitation, health education, food and water hygiene. Some were paid monthly wages of about US $\$ 20$ by the village councils, others worked on a voluntary basis, but the majority claimed a regular government salary. So far there is no experience regarding the use of VHW in leprosy or tuberculosis control measures. However, wherever VHW are working it is possible to train them to perform specific leprosy control tasks. The ministry of health has now prepared a uniform syllabus for VHW and will coordinate their activities. This decision is partly based on the intention to provide health for all by the year 2000 and partly on the realization that it will take 60 years at the present rate of expansion to provide conventional dispensaries and rural health centres to villages without any health facility. ${ }^{13}$

\section{Potential of PHC for achieving the goals of leprosy control}

Leprosy control measures recommended by WHO are early case-finding, surveillance of contacts, effective and prolonged chemotherapy and disability prevention. ${ }^{2,3}$ PHC has the potential for improving the approaches for casefinding. The main method of case-finding depends on the passive approach of examining motivated patients who visit health facilities. Active case-finding mainly through surveillance of contacts of multibacillary index cases is also important in increasing detection rates.

The passive approach depends on community awareness about leprosy. This awareness can be promoted when PHC educates the community on the major endemic diseases (leprosy being one of them) and on methods of 
prevention and control. Success here depends on having a person who speaks the people's 'language' and is capable of 'bridging intellectual and social distances, removing cultural barriers and, if necessary, changing attitudes and habits'. ${ }^{14}$ The VHW can also conduct contact surveillance more easily than other visiting health workers because of the knowledge he has about the villages, the trust they have in him and the fact that he is geographically more accessible to the villagers than health workers from distant health units. Under such circumstances early case-finding becomes a feasible prospect.

PHC can also promote early and effective chemotherapy. The first requirement for early drug treatment is early detection of the patients. It is also important that the patient should have the right attitude towards modern medicine vis-à-vis traditional medicine.

Even when patients are detected early and they are started on chemotherapy, there are other problems that must be overcome. It is generally accepted that presently available control methods require improvements because few, if any, of the developing countries where leprosy is still a major problem have achieved control of the disease. ${ }^{15}$ According to Browne ${ }^{16}$ the main reason for this is that many patients in any country do not receive chemotherapy long enough or regularly enough to interrupt the chain of transmission. Regular clinic attendance of $70-80 \%$ in the first year is achieved by control programmes but attendance quickly drops to even less than $20 \%$ after $3-4$ years. ${ }^{15}$ Although health education of the patient is very important, experience has shown that it is generally not sufficient to motivate patients to take drugs ${ }^{14}$ and it is not expected to have an important and rapid impact because it does not get full participation of the cummunity. ${ }^{3}$

PHC as presently defined addresses itself to these problems. It favours early treatment which not only benefits the individual patient but also protects healthy people by reducing the period during which they are exposed to infectious sources of viable bacilli. There is no other way of protecting healthy people except through early and effective treatment. Primary prevention with a vaccine is still in experimental stages. Isolation of patients is out of date and irrelevant because contacts are at greatest risk before the start of treatment. PHC brings treatment to the village of the patient, thus reducing the distance and time the patient has to travel to collect drugs. The major weapon for control, namely, case-finding/treatment, is therefore geographically and functionally made accessible to the community. PHC allows close supervision of patients and facilitates defaulter tracing, and thereby assures maximum regularity. It also arouses community interest in the control measures. ${ }^{5}$

Another objective of leprosy control is to prevent disability in new patients and to reduce it to the minimum among patients on treatment. This objective depends partly on the achievements of early detection through which patients are diagnosed before the disease has advanced and produced irreversible tissue damage. It also depends on the adequacy of the treatment. Health education 
which is given to patients has greater potential for reducing disability among patients with reversible disabilities than reconstructive surgery. ${ }^{17}$ VHW can be trained on methods of disability prevention. The achievement of this objective has far-reaching consequences, such as preserving the productive ability of patients, prevention of the disfigurement produced by leprosy and the effect of this in reducing the stigma of the disease.

\section{Present and future use of PHC in leprosy control in Tanzania}

A great deal of work must be done in Tanzania to make a significant impact on the leprosy problem. Although accurate prevalence data are still not available, a rational estimate puts the prevalence for the whole country at 6 per 1,000 population. ${ }^{18}$ Table 2 presents data of the patients on register at the end of 1979. The overall case detection rate was 35 per 100,000 population. Although most regions reported regular clinic attendance of between 60 and $70 \%$, the reliability of some of the data is highly questionable, making the true regularity even lower than the reported one. In contrast to our performance so far of detecting about $50 \%$ of the estimated multibacillary cases, WHO recommends that in order to achieve significant reduction in incidence, at least $75 \%$ of the infectious cases should be detected and receive regular treatment. ${ }^{15}$ That achievement will require more than the conventional health infrastructures and manpower.

Table 2. Annual statistics of leprosy patients at the end of 1979, Tanzania

\begin{tabular}{|c|c|c|c|c|c|c|}
\hline \multicolumn{2}{|c|}{ New registrations } & \multirow[t]{2}{*}{$\begin{array}{c}\text { Case } \\
\text { detection rate* }\end{array}$} & \multicolumn{2}{|c|}{$\begin{array}{c}\text { Total registered } \\
31.12 .79\end{array}$} & \multicolumn{2}{|c|}{$\begin{array}{l}\text { Regular } \\
\text { attendance rate }\end{array}$} \\
\hline BL-L & All cases & & BL-L & All cases & BL-L & All cases \\
\hline 1,119 & 5,661 & 35.2 & 11,601 & 48,662 & $71.8 \%$ & $70.6 \%$ \\
\hline
\end{tabular}

It must be remembered that leprosy is only 1 of the many health problems facing the country. One approach to maximizing the limited resources is to combine leprosy and tuberculosis services in Tanzania. This makes it possible to utilize transport, microscopes, laboratory reagents and supervisory staff at the central, intermediate and peripheral levels to co-ordinate the control measures against the 2 diseases. However, the most important approach is to implement PHC on a nation-wide scale.

The implementation of the National Tuberculosis and Leprosy Programme specifies that the control measures must be applied throughout the country. 
The data presented in preceding paragraphs showed that leprosy control in Tanzania cannot be carried out by professional staff in specialized units. The staff working in rural health centres, rural dispensaries and auxiliaries in hospitals must be involved in order to obtain country-wide coverage. However, the involvement of all these conventional health facilities extends the care to the place of residence of only $45 \%$ of the rural inhabitants. It is, therefore, necessary in the near future to utilize VHW in specific control measures for the remaining $55 \%$ of the rural population who do not have any health facility within the community.

In leprosy control the VHW should be able to recognize leprosy lesions and to refer the suspect for diagnosis, classification and prescription of the medicine. 'If there is 1 diagnosis that should not be established unless there is absolute certainty, it is that of leprosy.' 15 Once the drugs are prescribed, the VHW should be able to dispense the correct number of tablets for any given period and keep simple, accurate records which should enable him to detect irregular patients and trace them. He should be able to provide routine treatment for ulcers. With on-the-job training he should be able to give advice on the care of insensitive feet and hands and refer other complications. Contact surveillance, with emphasis on contacts of the multibacillary index cases, is another task which village health workers can perform. To facilitate supervision and on-the-job training VHW should have fixed clinic days. In Tanzania experience with workers in the village health posts showed that it is difficult to retain them on a purely voluntary basis. Hence some remuneration for them must be considered.

Adequate supply of drugs and equipment must be provided. Shortages of medicines should be avoided by providing adequate funds and by careful attention to the logistics of supplies. Lack of medicines in village health posts in Tanzania was given as the main reason why villagers under-utilized these facilities. ${ }^{13}$ In 1979 periodic shortages of dapsone were cited by health workers as a cause of irregular attendance of leprosy patients in a number of districts in Tanzania. It is therefore of crucial importance that supplies are available without interruptions.

A functioning referral system is indispensable to PHC in leprosy control. Tasks requiring better-trained workers include diagnosis and classification, decision about dosage of drugs for new patients, taking skin smears, review of clinical activity of the disease and discharging patients. More complicated problems such as lepra reactions and eye problems should be referred to hospitals. Tanzania's experience with VHW shows that for the task of treating minor ailments the VHW managed $87 \%$ and referred $13 \%$ of the daily average workload of 43 patients. ${ }^{13}$ The referral system should be organized in such a way that the district leprosy co-ordinator visits PHC clinics regularly to perform tasks for which patients would have to travel to the larger centres.

Effective supervision is of vital importance in leprosy control in peripheral 
areas. Regional tuberculosis/leprosy co-ordinators in Tanzania repeatedly underscored the importance of supervision in their annual reports for 1980 . Mulder (1980) ${ }^{19}$ visited each of the five districts in Shinyanga region nine times in 1980 to try to discover the magic key to better performance. He concluded that supervision by district co-ordinators must receive the highest priority. Van Deun (1980) ${ }^{20}$ in Kigoma region, while noting that adequate clinic supervision requires motivation, a lot of effort and courage, concluded that it was the most important part of control work because in its absence health units did not perform well. Experienced field workers have also pointed out that there are risks in delegating specialized activities to undertrained auxiliaries. There are risks of carelessness, indifference and occasional frank dishonesty ${ }^{21}$ in carrying out tasks such as collection and examination of specimens, recording and reporting. If supervision is of such vital importance with relatively well-trained auxiliaries, it will be indispensable with VHW who get only limited training. When community participation in PHC is adequate, the community can exercise managerial control of activites while supervisory health personnel can provide technical guidance and support to VHW. ${ }^{1}$

This mutual co-operation between the community and health workers in insuring proper implementation of health measures is not only an indicator of active community participation, but it may also hold the key to the realization of the full potential of PHC in health improvement in general and in leprosy control in particular.

\section{Acknowledgements}

I wish to thank Dr A D Chiduo, Minister of Health, for scrutinizing the draft and giving valuable criticisms and advice and for granting permission for publication.

I also wish to thank Dr D W Mulder, Tuberculosis/Leprosy Control Unit, Ministry of Health for his useful criticisms and suggestions.

\section{References}

Alma-Ata, Primary Health Care, WHO/UNICEF, 1978, 53-73.

2 WHO Technical Report Series No. 607, 1977 (5th Report of the Expert Committee on Leprosy).

${ }^{3}$ Sansarricq H. Review of Present Trends in Leprosy Control, presented at WHO Consultation Monrovia, Liberia, 1979.

4 Integration of Leprosy Services and Tuberculosis Services: Report of a Consultation, Monrovia, Liberia, 1979, WHO Regional Officer for Africa, Brazzaville.

${ }^{5}$ Buchmann H. Leprosy Control Services as an Integral part of Primary Health Care in Developing Countries, Germany Leprosy Relief Association, Wurzburg, 1978. 
${ }^{6}$ Mahler HT. Health for All by the year 2000, address made in Tokyo, Sasakawa Memorial Health Foundation, 1980; 7.

7 Guilbert J-J. Educational Handbook for Health Personnel, WHO, Geneva, 1977; 114.

${ }^{8}$ Chuke PO. The Relevance of Present Medical Training in Relation to Health for All by the year 2000, World Health Day 1981, AFRO/WHO/6.

9 WHO LAB/79.1. Laboratory Services at Primary Health Care Level, WHO Chronicle, 1979; 33: 334 .

10 Mkumbwa ZM. The Training of Primary Health Workers in Delivery of Primary Health Care 1979 (unpublished document).

11 Planning Unit, Ministry of Health, Health Care Planning in Tanzania, 1981 (unpublished document).

12 King M. Medical Care in Developing Countries, Oxford University Press, Nairobi, 1967; 2.6.

13 Planning Unit, Evaluation of the Health Sector, Ministry of Health, Tanzania 1979; $14 \& 143-51$.

14 Toman K. Tuberculosis Casefinding and Chemotherapy, What is the significance of default in the chemotherapy of tuberculosis? 1979; WHO, Geneva, 215.

15 WHO, A Guide to Leprosy Control, WHO, Geneva, 1980.

16 Browne SG, 1973 cited in Buchmann H. Leprosy Control Services as an Integral Part of Primary Health Care in Developing Countries, 1978; 11.

17 Smith WCS, Antin MS, Patole AR. Disability in Leprosy: a relevant measurement of progress in Leprosy Control. Lepr. Rev. 1980; $51: 155$.

18 A Guide for the National Tuberculosis/Leprosy Programme in Tanzania, Ministry of Health (in press).

19 Mulder DW. National Tuberculosis/Leprosy Annual Report, 1980 for Shinyanga Region, Tanzania (unpublished).

20 Van Deun A. National Tuberculosis/Leprosy Annual Report, 1980 for Kigoma Region, Tanzania (unpublished).

21 Fox W. Organizational and administrative considerations in the diagnosis and treatment of tuberculosis in developing countries. Tubercle, 1968;49: 332. 